\title{
An Improved Enhancement Technique for Cardiovascular Ultrasonic Image Analysis based on DCNN
}

\author{
Damodharan D ${ }^{1}$, Amit Kumar Goel ${ }^{2}$, Tapas Kumar ${ }^{3}$ \\ ${ }^{1}$ Research Scholar \& Assistant Professor, SCSE,Galgotias University, India \\ damodharan@galgotiasuniversity.edu.in \\ ${ }^{2}$ Professor,SCSE,Galgotias University, India, amit.goel@galgotiasuniversity.edu.in \\ ${ }^{3}$ Professor, CSE, Manav Rachna University Haryana, India ,tapaskumar.fet@ mriu.edu.in
}

\begin{abstract}
In medical field images processing techniques are play an important role to diagnosis the cardiovascular diseases. It truly can merit a patient's life at most time. Computerized reasoning and $\mathrm{AI}$ technique and devices are giving another chance to anticipate side effects a prior stage to conclusion. A proposed technic to implement the image enhancement method using filter and AI convolution neural network for the training and testing cardiovascular ultrasonic image. This paper is described through over the ongoing patterns and procedures to finding and whether a person has cardiovascular disease. For a Proposed work applying the medical re enhancing the frame developed the good accuracy $(97.33 \%)$
\end{abstract}

Key words: Cardiac Imaging Techniques, Diagnostic Imaging, Echocardiography, Image Enhancement, Tomography, Ultrasonic.

\section{INTRODUCTION}

Cardiovascular disease is present leading one of the deadly cases all around the world. It follows expected the improvement from computing techniques that can forecast the closeness of coronary illness will basically reduce coronary illness caused death rate, Although early location could prompt considerable to decrease care investment prices. Traditional statistical techniques describe illations from a set of factors acquired tests executed below ensured conditions.

Approximately 7.8 lakes people are affected and 2.5 lakes people are die every year on cardio arrest within every 60 minutes onset of indications of body activity and earlier he contact hospitals[3]. Recently attempts to create computational models are fit for investigating and anticipating whether a person has coronary diseases have demonstrated extraordinary promise [1]. The target of this work was to make classifiers to promise whether somebody has cardiovascular diseases illness. Echocardiograms are a difficult preparing dataset, in any case, since one ultrasound study contains of still pictures and videos caught from over twelve points. The data sets were obtained from different university are providing online free sources provided for research purpose like university as Cleveland Clinical Organization and Hungarian Intuition of Cardiology from different country. Some data sets are taken online website support like kaggle, thepocusatlas, etc...

The database comprises of medical screening results and cardiovascular disease diagnoses for 600 individuals. Works are done by into two part. Part I based on data reprocessing and Model establishing and screening will be presented in Part II. The outcome are summarized in the concluding section. Ultrasonic technology they include echo cardiograph (echo) TTE - Transthoracic echo, TEE-Transesophageal echo, ultrasound software and POC point of care are all is an invasive form of cardiac ultrasound (echo) wave.

Type of heart Diseases

- Abnormal Wall Motion

- Heart Failure

- Valvular Heart Disease

- Atherosclerosis

- Coronary Artery Disease

- Cardiomyopathies

- Myocardial Infarction

\section{MATERIALS AND METHODS}

\subsection{Method and equation and architecture}

The Table 1 shows various methods used for various stages and for the comparative study for the machine learning model and Logistic regression denote a linear regression that product output signal of a binary classification. Irrespective of tool $\mathrm{R}$ is going to work on to calculate the accuracy of the model with linear equation

$$
G(E(y))=a+b x_{1}+c x_{2}
$$


Damodharan D et al., International Journal of Advanced Trends in Computer Science and Engineering, 9(5), September - October 2020, 7087- 7091

Where equation (1) provide in details about $\mathrm{G}(\mathrm{E}(\mathrm{y}))$ is the connection function, $\mathrm{E}(\mathrm{y})$ is the arithmetic.
The Figure 1 shows Wait of $\mathrm{w} 1, \mathrm{w} 2 \ldots \mathrm{w}_{\mathrm{n}}$ for the classification from ANNs is an uncomplicated mathematical model to increase surviving data analysis technologies [15]. Still it is the basic building block of Artificial intelligence model.

Table 1:Comparative study between passive and active systems.

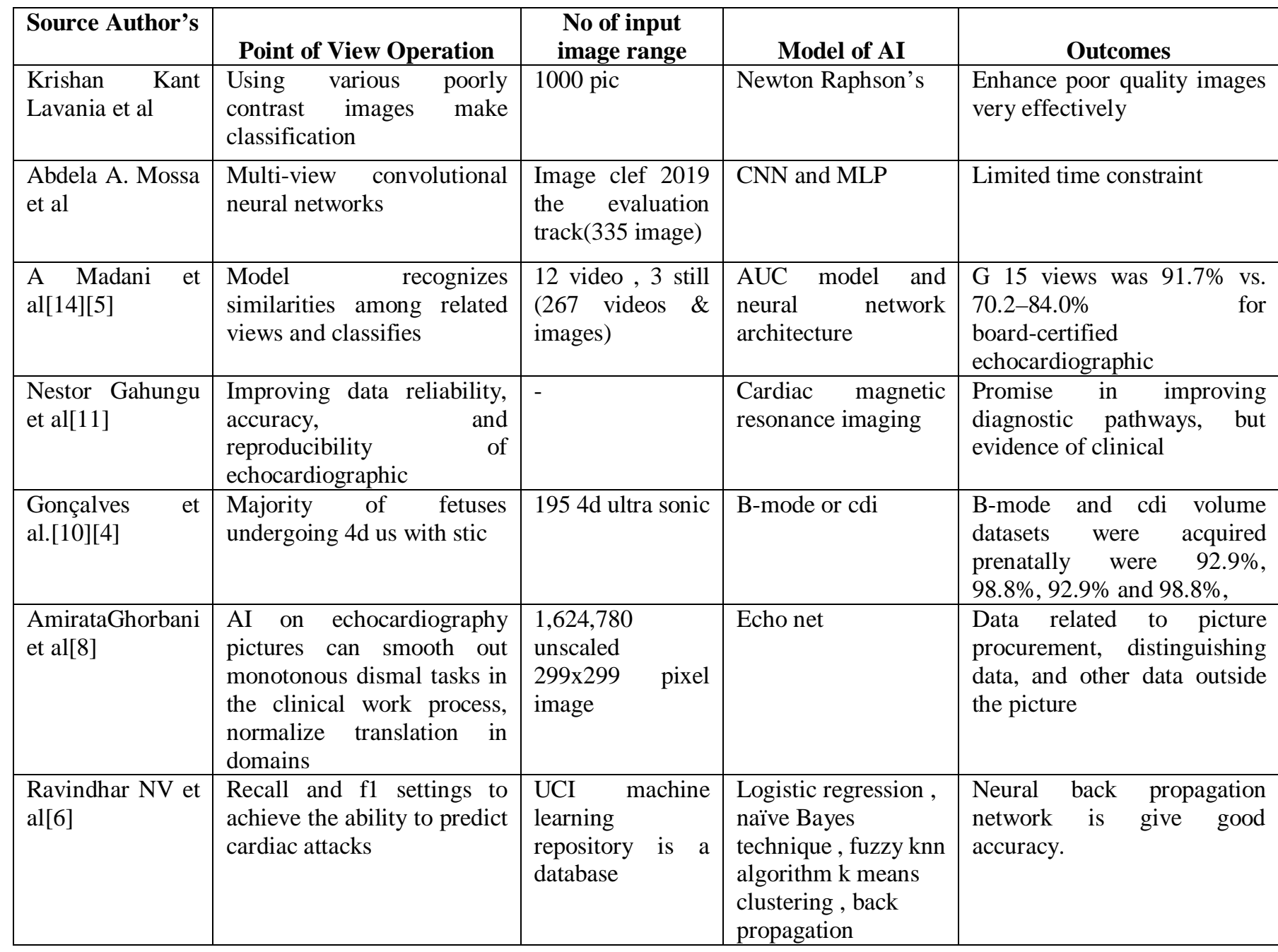

mean of pointed variable and the linear predictor is $a+b x 1$ $+\operatorname{cx} 2$ (a, b,c to be predicted). A purpose of connection function is to 'link' the prospect for y to linear predictor. Calculate the model Accuracy is:

$$
\text { Accuracy }=\frac{T P+T N}{T P+T N+F P+F N}
$$

Support Vector Machine (SVM) Finds the optimal boundary between classes for standardization such that $w>x++b=+1$, $\mathrm{w}>\mathrm{x}-+\mathrm{b}=-1$ for the Negative and Positive support vectors severally. Hence $w>x+b=0, c$ $(\mathrm{w}>\mathrm{x}+\mathrm{b})=0$ the very well in the same skim, we have the exemption to select the normalization of w. Artificial Neural Network Model is a composite classification tasks by circulating the input information done a network of non-linear transformations.

$$
f(x)=\sum_{i} a_{i} y_{i}\left(\boldsymbol{X}_{i}^{T} \boldsymbol{X}\right)+b
$$

$$
\begin{aligned}
z= & f(x . w)=f\left(\sum_{i=1}^{n} x_{i} \cdot w_{i}\right) \\
& \mathrm{x} \in \mathrm{d}_{1 * \mathrm{n}}, \mathrm{w} \in \mathrm{d}_{\mathrm{n} * 1}, z \in \mathrm{d}_{1 * 1}
\end{aligned}
$$

The equivalence for an established nodes look as follows. The weighted add together its inputs extended through a non-linear activation methods. It tends to be spoken to as a vector spot item, where $\mathrm{n}$ is the quantity of contributions for the node.

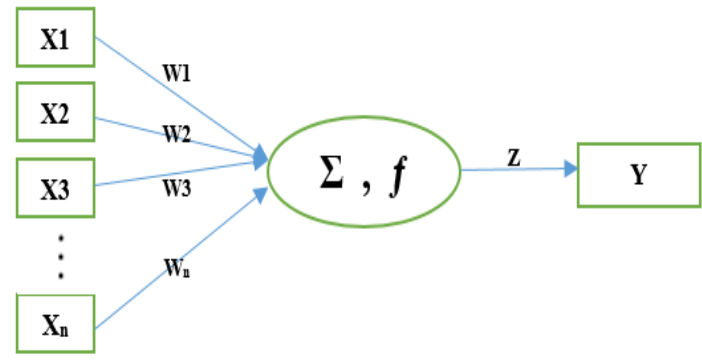

Figure 1: Artifact Neural Networks with Wait for the classification 
A neural framework is an AI computation subject to the model of a human neuron. The human cerebrum includes an enormous number of neurons. It grants and procedure signs as electrical and engineered signs. These neurons are related with an uncommon structure known as synapses. Synapses license neurons to transmit signals. From colossal amounts of reproduced neurons frameworks structures.

An ANN is information take care of Numerical technique. It works like the way where human brain structures information. ANN joins a huge number of related taking care of units that coordinate to process information. They furthermore make noteworthy results from it. For the practice neural framework is not just for classification system. It can in like manner apply for backslide of unending target qualities.

Convolutional Neural Network (CNN) ANNs balanced for the preparing and characterization of picture.

The Figure 2 shows an implementation of the working model A Convolutional Neural Network is an advanced technique to calculate a Deep Learning figuring which can take in a pictures information, consign essentialness to alternate points of view/inquiries in the image and have the choice to isolate one from the other. The pre-taking care of required in a CNN is a great deal of lower when appeared differently in relation to other request counts. While in unrefined procedures channels are hand-planned, with enough getting ready, ConvNets can get acquainted with these channels/characteristics.

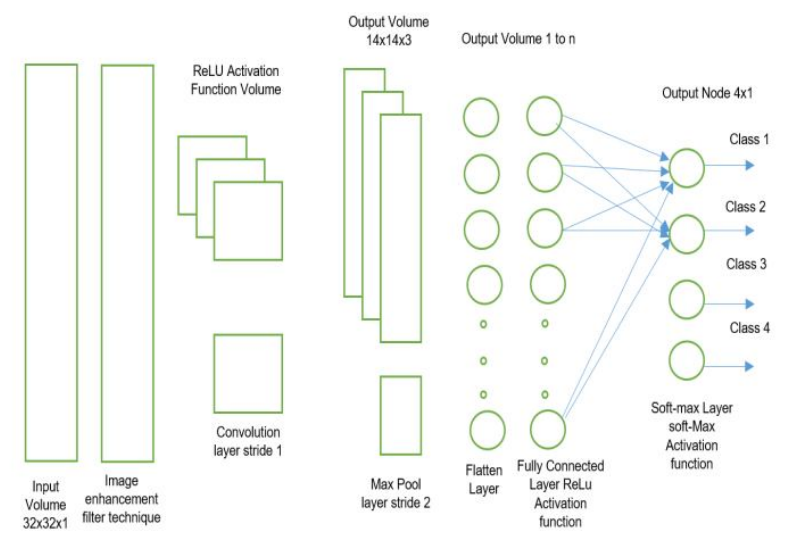

Figure 2:Enhancement with Deep convolution neural network

There are different models of CNNs open which have been key in building computations which power and will power AI in general within a reasonable time-frame.

\section{PROPOSED METHODS}

\subsection{Heart diseases Basic Characteristic factors}

The risk factors for heart disease include the following:

- Age in Years

- Gender(Male and Female)

- Chest Pain Variety(4 types)

- Thest bps(Blood Forse while slipping)

- Cholesterol Serum
- Blood Sugar $(1>=120 \mathrm{mg} / \mathrm{dl}, 0<=120 \mathrm{mg} / \mathrm{dl})$

- Etc...

$\mathrm{X}$ : The input information characterized to $\mathrm{X}$ above. It carries the $\mathrm{x}$ and $\mathrm{y}$ directions of the input directions.

Y: Not to be mistaken for the y facilitate of the information focuses. In all ML instructional exercises y alludes to the names, for our situation the class we're attempting to prediction: 0 or 1 .

Verbose: Prints out the misfortune and exactness, set it to 1 to see the yield.

Ages: Possible to find all the times to go over the whole preparing information. When preparing models we go through the preparation information once as well as numerous occasions. And Figure 3 Procedure of building up a clinically significant to training and testing for using AI model and related difficulties in echocardiography.

\subsection{Proposed Algorithm}

Step 1: Collect the set of inputs $X$ and divide the same in $n$ classes where each $\mathrm{x}$ has $\mathrm{A}$ attributes and a have a specific target value ' $t$ '.

Step 2:-Find the decision boundaries of each class \& attribute Calculated the training

Positive dataset $P P=\left\{a 1, a 2, a 3, a 4 \ldots a_{n}\right\}$, Negative dataset $P N=\left\{b 1, b 2, b 3, b 4 \ldots b_{n}\right\}$.

Step 3: Initialization set value $\mathrm{i}$

Step 4: check the no of iteration and trial $t$ until condition failt $=$ Trial, set the subset $\mathrm{I} \square$ of $\{1,2 \ldots \mathrm{N}\}$

, J of $\{1,2 \ldots \mathrm{M}\}$ Construct the air with $\mathrm{q}$

Step 5: using the Filter to enhancement and update pi

Step 7: the above steps will generate new data sets

Step 8: End Return net Layer and classification machine learning algorithm.

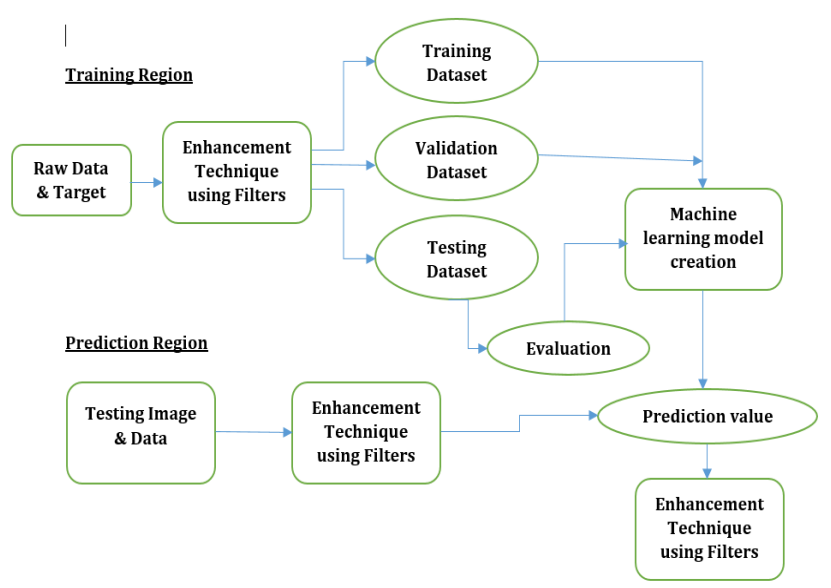

Figure 3:Procedure of building up a clinically significant AI model and related difficulties in echocardiography.

They assured the suitable number of layer and its filter for channel counts are underlying phase from the present concentrate with they chose associate [4]. In this stage, the 
found the middle value of picture was utilized. The partner included 600 patients, which was partitioned as $(780,250$, 250) for (train, approval, test), individually. In the preparation, the cross-entropy mistake work was utilized as the misfortune work.

We utilized the ADAM streamlining agent with the default parameters aside from an underlying learning pace of 0.0005 and group size of 5 . The quantity of the ages was set as 50 . The model loads were put away when the approval misfortune was limited. The precision of the view characterization for the test partner was utilized in the assessment.

\section{RESULTS AND DISCUSSION}

Classification is a fundamental improvement toward the translation of echocardiographic pictures in a clinical backgrounds. We tried an AI model that accurately arranged customary echocardiographic information to various perspectives in the screening accomplice. The try-out result associate information constituted assembled from back to back clinical datasets got for clinical strategy, from patients with a wide degree of ages, sizes, and hemodynamics. In like manner, stunningly after the utilization of learning information with a $0.5 \%$ blunder rate, the precision of the echocardiogram want model for TEE was legitimized. This model is a possible picture game-plan system. In diagram, our evaluation showed $97.2 \%$ when in doubt test precision of a CNN calculation in the free accessory, and $98.33 \%$ of tests should be exactly separate to make a plausible clinical check model of T. A past report (Madani et al.) [16] Used models subject to a thin scope of Contrasted with this past examination, our examination included patients with a wide arrangement of systolic limits, recorded by machines be that as it may, the exactness of our opinion characterization method was sufficient to make ye forecast method for CNN. Advance examination model wanted to investigate the adequate error rate. It didn't contrast the AI models and other AI\&ML models in this examination.

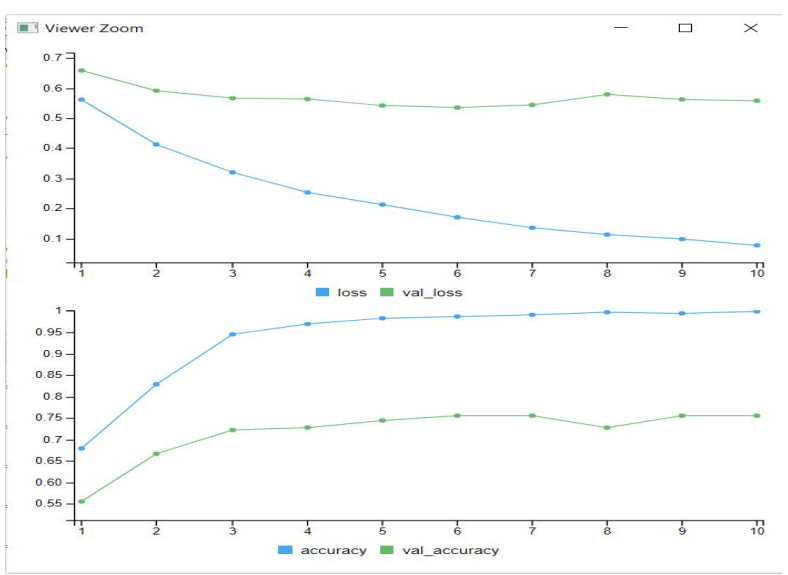

Figure 4:Graph for Testing and Accuracy \& Loss Rate

In our past-II examination on expectation of an Images [9], we utilized an element descent strategy, comprising of cross connection and optical stream. In the outcomes, the element decline strategy existed appropriate to foresee the TEE utilizing echocardiographic pictures contrasted and CNN models Along these lines, they chose to apply the proposed model for echocardiographic see order in this examination. Our outcomes affirm on a fundamental level that CNN might be viable in the view arrangement, yet bigger quantities of patients ought to be assessed to gauge the viability of programmed determination frameworks in a clinical setting. It is concluded from the graph that alone passive or active systems are not appropriate and sustainable due to increasing energy demand trend in space heating/cooling. It forces us to adopt suitable hybrid systems according to tailor made situations.

\section{CONCLUSION}

Ultrasonic recognition of carotid plaque and CIMT estimations can be helpful for refining CVD chance evaluation in some asymptomatic patients. Exacting regard for quality control in picture securing, estimation, understanding, and revealing are vital for usage of this method in clinical practice. Computer based intelligence is promising to change a wide range of cardiovascular imaging, including echocardiography. The essential clinical points of interest of ML counts in echocardiography will recollect decline for time spent isolating and consolidating echocardiographic data and improvement in reproducibility of echocardiographic evaluations. Other potential focal points will consolidate joining of Machine Learning into chance gauge methods and early area of cardiovascular contaminations at preclinical stages.

\section{FUTURE SCOPE}

In future, we can collect a large amount of data set and create a diagnosing tool in AI based R, Python which can help doctors in early detection of heart disease and proper treatment of the heart disease.

Information Availability the information supporting this audit are acquired from recently revealed investigations and datasets, which have been referred to inside the composition as references.

\section{REFERENCES}

1. Shameer K, Johnson KW, et al. Heart. 1-9, 2018.

2. Boon N, Norell M, Hall J, Jennings K, Penny L, Wilson $\mathrm{C}$, et al. National variations in the provision of cardiac services in the United Kingdom: second report of the British cardiac society working group, 2005. Heart. 2006;92(7):873-8.

3. Kukar M, Kononenko I, Grošelj C, Kralj K, Fettich $\mathrm{J}$.Analysing and improving the diagnosis of ischaemic heart disease with machine learning.ArtifIntell Med. (1999) pp. 25-50.

4. Narula S, Shameer K, Salem Omar AM, Dudley JT, Sengupta. Machine-learning algorithms to Automate morphological and functional assessments in 2D echocardiography.J Am CollCardiol. 2016;68(21):pp. 2287-95.

5. Sengupta PP, Huang YM, Bansal M, Ashrafi A, Fisher M, Shameer K. Cognitive machine-learning algorithm 
for cardiac imaging: a pilot study for differentiating constrictive pericarditis from restrictive cardiomyopathy. CircCardiovasc Imaging. 2016;9(6).

6. Ravindhar NV, Anand, Hariharan Shanmugasundaram, Ragavendran, Godfrey Winster. Intelligent Diagnosis of Cardiac Disease Prediction using Machine Learning International Journal of Innovative Technology and Exploring Engineering (IJITEE), Volume-8 Issue-11, September 2019

7. Martin-Isla $\mathrm{C}$, Campello VM, Izquierdo $\mathrm{C}$, Raisi-Estabragh Z, Baeßler B, Petersen SE and Lekadir K. Image-Based Cardiac Diagnosis With Machine Learning: A Review. Front. Cardiovasc. Med. 7:1.

8. Amirata Ghorbani, David Ouyang, Abubakar Abid, Brya $\mathrm{n}$ He, Jonathan H. Chen, Robert A. Harrington, David H. Liang, Euan A. Ashley, James Y. Zou Deep Learning Interpretation of Echocardiogramsbio Rxiv 681676.

9. Azira Khalil,Siew-Cheok Ng, YihMiinLiew, Khin Wee Lai.An Overview on Image Registration Techniques for Cardiac Diagnosis and TreatmentHindawi Cardiology Research and Practice Volume 2018, Article ID 1437125, 15 pages.

10. Luís F. Gonçalves, Jimmy Espinoza, Roberto Romero, Juan Pedro Kusanovic, Betsy Swope, JyhKaeNien, Offer Erez, Eleazar Soto, and Marjorie C. TreadwellFour-Dimensional Ultrasonography of the Fetal Heart using a Novel Tomographic Ultrasound Imaging Display , J Perinat Med. 2006 pp. 39-55.

11. Nestor Gahungu, Robert Trueick \& Saiuj Bhat \&ParthoP. Sengupta, Girish Dwivedi, Current Challenges and Recent Updates in Artificial Intelligence and Echocardiography Current Cardiovascular Imaging Reports. (2020).

12. Zhang J, Gajjala S, Agrawal P, Tison Geoffrey H, Hallock Laura. A, Beussink-Nelson L, et al. Fully automated echocardiogram interpretation in clinical practice. Circulation. 2018. pp.1623-35

13. Gahungu, N., Trueick, R., Bhat, S.Current Challenges and Recent Updates in Artificial Intelligence and Echocardiography. CurrCardiovasc ImagingRep 13, 5,2020 .

14. Madani, A, Arnaout, R,Mofrad, M. Fast and accurate view classification of echocardiograms using deep learning. NPJ Digit. Med. 2018.

15. NS Kumar, $\mathrm{K}$ Anandhan, $\mathrm{S}$ Kumar. A Review on Spotting and Segmenting The Tumor Using MammographyJournal of Innovation in Computer Science and Engineering, 2019, Vol 4 pp.29-33.

16. Kusunose K, Abe T, HagaA, Fukuda D, Yamada H, Harada M. A deep learning approach for assessment of regional wall motion abnormality from echocardiographic images. JACC Cardiovasc Imaging. (2019).

17. http:// bocaradiology.com 